\title{
Kinesin and Myosin Motors Compete to Drive Rich Multi-Phase Dynamics in Programmable Cytoskeletal Composites
}

\section{Daisy Achiriloaie}

W. M. Keck Science Department, Scripps College, Pitzer College, and Claremont McKenna College Christopher Currie

University of San Diego

Jonathan Michel

Rochester Institute of Technology

Maya Hendija

University of San Diego

K Alice Lindsay

Syracuse University

Nadia Schwartz Bolef

W. M. Keck Science Department, Scripps College, Pitzer College, and Claremont McKenna College Gloria Lee

University of San Diego https://orcid.org/0000-0002-0761-9127

Michael Rust

University of Chicago

Janet Sheung

W. M. Keck Science Department, Scripps College, Pitzer College, and Claremont McKenna College Moumita Das

Rochester Institute of Technology https://orcid.org/0000-0002-0397-8886

Jennifer Ross

University of Massachusetts Amherst

Ryan McGorty

University of San Diego https://orcid.org/0000-0002-6577-060X

Rae Robertson-Anderson ( $\nabla$ randerson@sandiego.edu )

University of San Diego https://orcid.org/0000-0003-4475-4667

\section{Article}

Keywords: 
Posted Date: January 6th, 2022

DOI: https://doi.org/10.21203/rs.3.rs-1179494/v1

License: (c) (i) This work is licensed under a Creative Commons Attribution 4.0 International License. Read Full License 


\title{
Kinesin and Myosin Motors Compete to Drive Rich Multi-Phase Dynamics in Programmable Cytoskeletal Composites
}

Daisy H. Achiriloaie ${ }^{1,2}$, Christopher J. Currie ${ }^{1}$, Jonathan Michel ${ }^{3}$, Maya Hendija ${ }^{1}$, K. Alice Lindsay ${ }^{4}$, Nadia M. Schwartz Bolef ${ }^{2}$, Gloria Lee ${ }^{1}$, Michael J. Rust ${ }^{5}$, Janet Y. Sheung ${ }^{2}$, Moumita Das ${ }^{3}$, Jennifer L. Ross ${ }^{4}$, Ryan J. McGorty ${ }^{1}$, Rae M. Robertson-Anderson ${ }^{1, *}$

${ }^{1}$ Department of Physics and Biophysics, University of San Diego, San Diego, California 92110, United States

${ }^{2}$ W. M. Keck Science Department, Scripps College, Pitzer College, and Claremont McKenna College, Claremont, California 91711, United States

${ }^{3}$ School of Physics and Astronomy, Rochester Institute of Technology, Rochester, New York 14623, United States

${ }^{4}$ Department of Physics, Syracuse University, Syracuse, New York 13244, United States

${ }^{5}$ Department of Molecular Genetics and Cell Biology, University of Chicago, Chicago, Illinois 60637, United States

*randerson@sandiego.edu

\begin{abstract}
The cytoskeleton of biological cells relies on a diverse population of motors, filaments, and binding proteins acting in concert to enable non-equilibrium processes ranging from mitosis to chemotaxis. The cytoskeleton's versatile reconfigurability, programmed by interactions between its constituents, make it a foundational active matter platform. However, current active matter endeavors are limited largely to single force-generating components acting on a single substrate far from the composite cytoskeleton in live cells. Here, we engineer actin-microtubule composites, driven by kinesin and myosin motors and tuned by crosslinkers, that restructure into diverse morphologies from interpenetrating filamentous networks to de-mixed amorphous clusters. Our Fourier analyses reveal that kinesin and myosin compete to delay kinesin-driven restructuring and suppress de-mixing and flow, while crosslinking accelerates reorganization and promotes actinmicrotubule correlations. The phase space of non-equilibrium dynamics falls into three broad classes - slow reconfiguration, fast advective flow, and multi-mode ballistic dynamics - with structure-dynamics relations described by the relative contributions of elastic and dissipative responses to motor-generated forces.
\end{abstract}

\section{Introduction}

The cytoskeleton is a dynamic, non-equilibrium material comprising of protein filaments, including actin, microtubules and intermediate filaments, as well as force-generating motor proteins, such as myosins and kinesins, that actively push and pull on the protein filaments ${ }^{1-8}$. Crosslinking proteins also connect and bundle filaments as needed for cellular processes ${ }^{9-12}$. This complex composite continuously restructures and reconfigures itself in response to the demands of the cell, to enable diverse processes from cytokinesis to mechano-sensing s $^{3,7,8,13-21}$. In vitro systems of reconstituted cytoskeletal proteins, which display rich and tunable dynamics, are also intensely studied as model active matter platforms to shed light on the non-equilibrium physics underlying force-generating, reconfigurable systems ${ }^{7,12,19,22-40}$.

Interacting networks of semiflexible actin filaments and rigid microtubules provide tensile and compressive strength to the cytoskeleton while allowing for cell mobility, key to processes such as division and chemotaxis ${ }^{15,16,41-45}$. Further, recent studies have shown that composites of coentangled and crosslinked actin and microtubules exhibit emergent mechanical properties that are 
not a simple sum of the single component systems ${ }^{46-48}$. For example, composites with comparable concentrations of actin and microtubules display enhanced filament mobility compared to singlecomponent systems while at the same time exhibiting increased stiffness ${ }^{46}$. Further, composites display a non-monotonic dependence of elasticity on actin crosslinking not seen in the absence of microtubules ${ }^{47}$.

More recently, myosin II minifilaments have been incorporated into co-entangled actinmicrotubule composites, showing that synergistic interactions between actin and microtubules prevent disordered flow and actin network rupturing that is often seen in actomyosin networks without crosslinkers ${ }^{26-28}$. These studies have also shown that composites with comparable concentrations of actin and microtubules display desirable emergent properties including enhanced mechanical strength ${ }^{27}$, coordinated motion of actin and microtubules, sustained ballistic contraction and mesoscale restructuring ${ }^{26,28}$ - all in the absence of crosslinking proteins to chemically connect the filaments.

Microtubule-based active matter systems have also been engineered using clusters of kinesin motors that can crosslink and pull on bundles of microtubules to create active nematics $23,24,30,31,34,35,49-55$. In these systems, kinesin clusters generate long lasting turbulent flows by cyclically extending, buckling, fracturing, and healing microtubule bundles ${ }^{49}$. More recently, entangled actin networks have been incorporated into these active MT fluids, resulting in turbulent-like flow, bulk contraction or formation of layered asters ${ }^{29}$.

The distinct dynamics and structures that kinesin-driven and myosin-driven systems display begs the question as to how these distinct active components cooperate or compete with one another to control non-equilibrium cellular processes. Nevertheless, current state-of-the-art active matter designs rely on a single force-generating component. While composite active matter systems are beginning to emerge as a means to introduce more control and tunability over single-substrate systems $^{26-29,56}$, the dynamics that arise from two active components that act on distinct constituents of a composite system represent a new paradigm in active matter. Once achieving this advance, one can also determine how the connectivity of the active and passive substrates can be used to tune the active dynamics and reorganization. Beyond the relevance to the cytoskeleton, answering these key questions will elucidate general design principles for programmable materials with nonequilibrium properties, force-generation, and reconfigurability; and determine how to enhance programmability and expand the phase space of dynamics and structures by altering the active and static nature of crosslinkers and the substrates on which they act.

Here, we engineer co-entangled composites of microtubules and actin filaments that undergo a rich combination of advective flow, contraction, and multi-mode restructuring driven by kinesin and myosin motors. These dynamics are coupled to distinct time-evolving structures that range from interpenetrating scaffolds of actin and microtubules to microscale phase-separated amorphous clusters. We couple differential dynamic microscopy (DDM) with spatial image autocorrelation (SIA) analysis and particle image velocimetry (PIV) to discover that competition between kinesin-microtubule activity and actomyosin activity delays the onset of rapid restructuring and enables the sustainability of a percolated double network. We further show that crosslinking of either actin or microtubules accelerates the time-evolution of active dynamics and enhances actin-microtubule interactions. Despite these complexities, we find that we can divide the broad phase space of active dynamics and reorganization into three general Classes defined by their distinct dynamics-structure relationships. 


\section{Results and Discussion}

Active cytoskeleton composite design and formulation space. We engineer composites of coentangled networks of microtubules and $\operatorname{actin}^{46}$ and incorporate kinesin clusters that can crosslink and push and pull on pairs of microtubules to generate force and motion ${ }^{49}$, much in the same way myosin II minifilaments crosslink and pull on actin filaments ${ }^{57}$ (Fig 1A). We investigate the effect of static crosslinking of the active (microtubules) or static (actin) networks using biotinNeutrAvidin crosslinking at crosslinker:protein molar ratios $R$ that are high enough to induce measurable changes in the viscoelastic properties compared to unlinked networks, but low enough to prevent filament bundling ${ }^{47}$. More importantly, we incorporate a second active component, myosin II minifilaments, to generate force and motion of the actin network at the same time as the kinesin motors act on the microtubule network (Fig 1A,B). We note that for all composite formulations we keep the respective concentrations of actin, tubulin, and kinesin fixed, and simply include or exclude myosin motors, actin crosslinkers, and/or microtubule crosslinkers. We show that these very subtle changes in connectivity and actomyosin activity lead to dramatic changes in the active restructuring, exhibiting emergent dynamics and a broad programmable phase space of non-equilibrium properties.
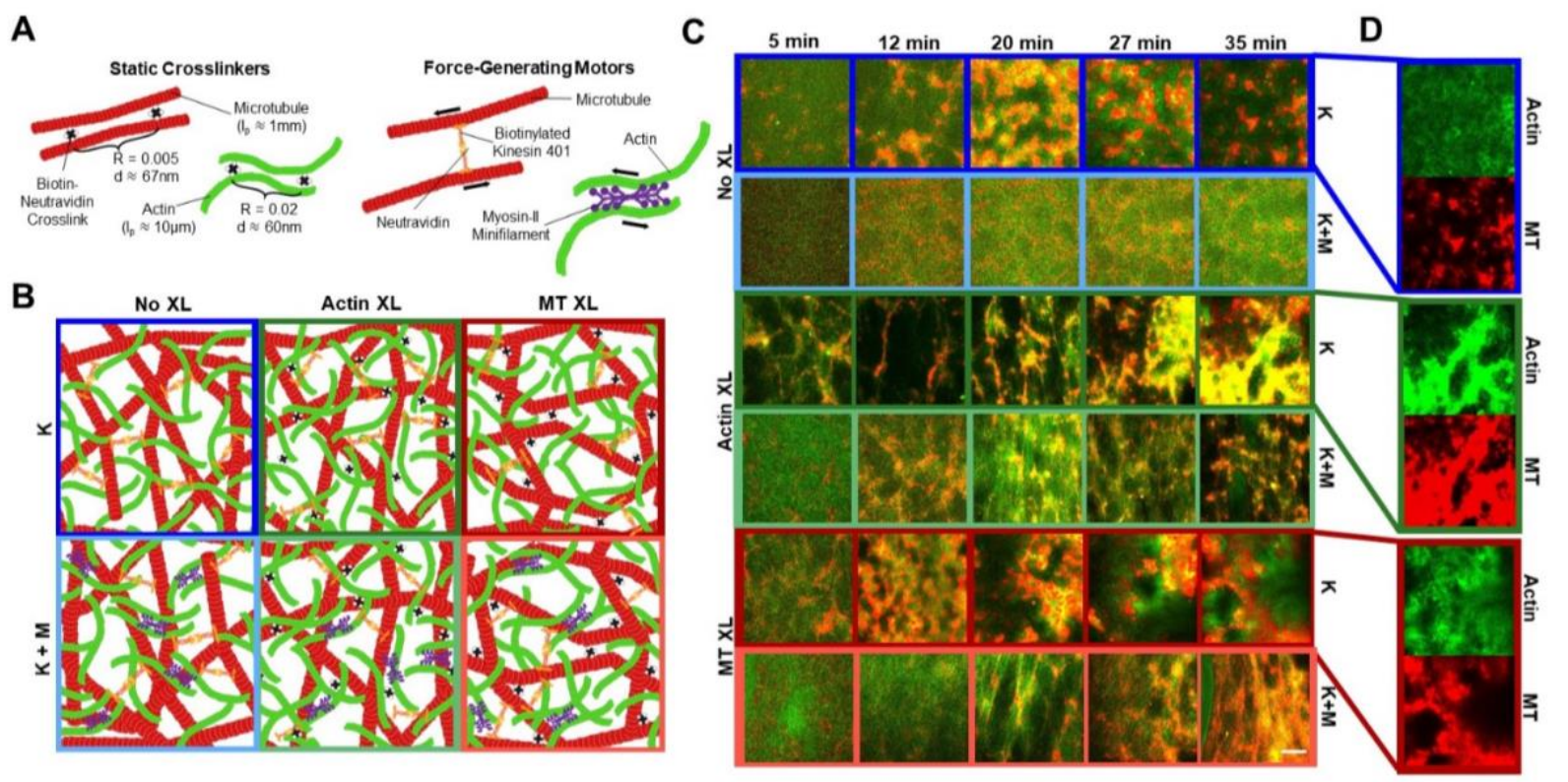

Figure 1. Engineering and characterizing active cytoskeleton composites with varying forcegenerating components and connectivity. A. We co-polymerize actin monomers $(2.32 \mu \mathrm{M})$ with tubulin dimers $(3.48 \mu \mathrm{M})$ to form co-entangled composite networks of actin filaments (green) and microtubules (red). Static crosslinking is achieved using NeutrAvidin to link biotinylated actin filaments (Actin XL) or microtubules (MT XL). The crosslinker to protein molar ratio $R$ is fixed at $R_{A}=0.02$ for actin and $R_{M T}=$ 0.005 for microtubules to achieve similar distances $d$ between crosslinks along the filaments ${ }^{48}$. We incorporate kinesin clusters (orange) and myosin-II minifilaments (purple) to generate forces in the composites and drive them out of steady-state. B. Cartoon of composite formulation space. We incorporate $0.35 \mu \mathrm{M}$ kinesin motors (K) into composites with no static crosslinkers (No XL, dark blue box), actin-actin crosslinks (Actin XL, dark green box) and microtubule-microtubule crosslinks (MT XL, dark red box). For each kinesin-driven composite, we also examine the effect of adding $0.47 \mu \mathrm{M}$ myosin motors $(\mathrm{K}+\mathrm{M})$ into composites with no static crosslinkers (No XL, light blue box), actin-actin crosslinks (Actin XL, light green 
box) and microtubule-microtubule crosslinks (MT XL, light red box). C. We use a Nikon A1R confocal microscope to acquire two-color time-series of the actin (green, AlexaFluor488-labeled) and microtubules (red, rhodamine-labeled) in the composites to capture the time-evolving reconfiguration and dynamics. Composite images, each from a different time-series, show complex formulation-dependent restructuring over time (listed in mins). Scale bar is $50 \mu \mathrm{m}$ and applies to all images. Outline colors match configurations shown in B. D. Separate actin and microtubule fluorescence channels show varied structures with both actin-MT co-localization and micro-phase separation across the formulation space.

Motor-driven reconfiguration is tuned by crosslinking. As shown in Fig $1 \mathrm{C}$, all composites begin in similar structural states with interpenetrating networks of actin and microtubules uniformly distributed throughout the field of view. However, the composites actively rearrange into distinct states dictated by the crosslinking motif on a timescale of minutes. Kinesin-driven composites without static crosslinkers form loosely connected amorphous clusters that are MT-rich. Actin colocalizes in the centers of these aggregates but over time they are squeezed out of the MT-rich regions into the surrounding space as the MT aggregates continue to contract and disconnect from one another (Fig 1C, dark blue boxes).

Introducing actin-actin crosslinking hinders this microscale actin-MT separation and instead the MT-rich pockets are connected via long strands of actin. Interestingly, actin crosslinking enables slow uptake of actin into the MT-rich regions rather than expulsion, such that the composite becomes a connected network of clusters of co-localized actin and microtubules (Fig 1C, dark green boxes). Microtubule crosslinking leads to similar amorphous clustering of MTs as in the case without crosslinking, but in this case, the MT-rich regions coalesce over time, resulting in larger scale phase separation of actin and MTs (Fig 1C, dark red boxes).

Given the complex structural differences that static crosslinking confers in kinesin-driven actinMT composites, we sought to determine the effect of a second independently tunable active component, myosin II, that acts on actin filaments rather than microtubules. As shown in Figure $1 \mathrm{C}$, the introduction of myosin arrests the demixing caused by kinesin alone, and causes the initial actin and microtubule networks to be even more evenly distributed and interpenetrating than the starting state of the composites without myosin. Further, in all cases, myosin surprisingly reduces the degree of restructuring rather than enhancing it (Fig 1C, light shaded boxes). Without static crosslinkers, the composites show little rearrangement over the course of a few hours, as seen in previous experiments on myosin-driven actin-MT composites ${ }^{26-28}$. Crosslinking of either the actin or microtubules leads to more network restructuring and increased co-localization of actin and microtubules compared to kinesin alone. Specifically, when microtubules are crosslinked, there is significant overlap of the networks and reorganization into web-like networks of fibers.

To quantify the reorganization shown in Fig 1, we compute the spatial image autocorrelation (SIA) of the actin and microtubule channels of images taken at different times over the course of activity (Fig 2). The autocorrelation $g(r)$ determines the degree to which the intensity at one location in the image correlates with the intensity of the surrounding points at varying distances $r$. The more quickly $g(r)$ decays, the smaller the structural features of the network.

Figure 2A shows autocorrelation curves for the actin and microtubule channels of the first and last frame of three representative $\sim 6$ min videos (SI Movies 1-3) that highlight the distinct network reconfigurability we observe. We divide these data into three broad classes. 'Class 1' shows limited spatial correlations that quickly decay over short length scales $(<10 \mu \mathrm{m}$, Fig $2 \mathrm{~A}$, top panel). 
'Class 2' shows stronger short-range correlations and a much weaker decay with length, persisting across the entire field of view (Fig 2A, middle panel). 'Class 3' data exhibit the strongest shortrange correlations and a decay that is in between that of Class 1 and Class 3 (Fig 2A, bottom panel).
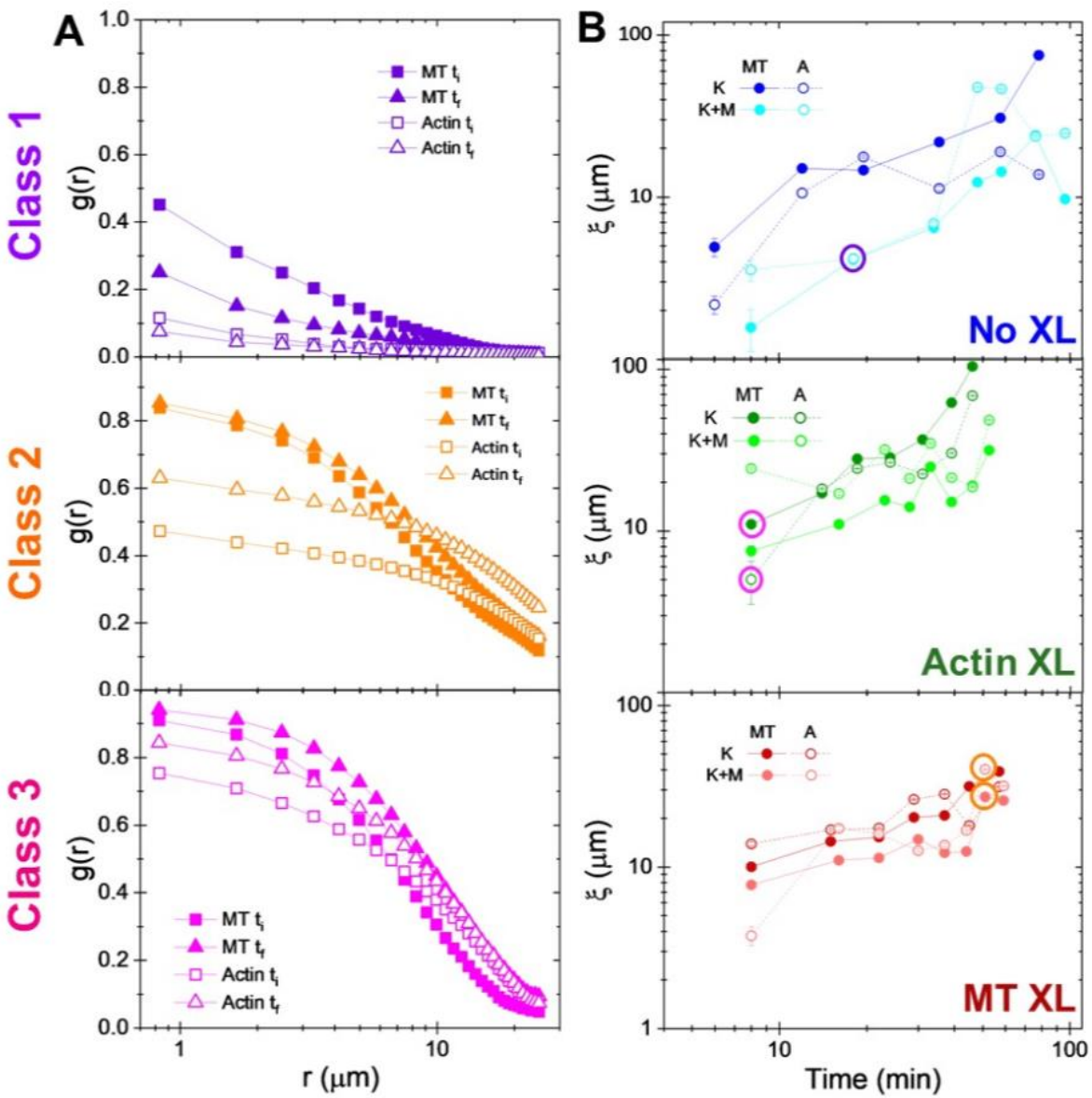

Figure 2. Spatial image autocorrelation analysis reveals distinct classes and reconfigurability of kinesin-driven composites. A. Radially averaged autocorrelation functions $g(r)$ vs separation distance $r$ computed for microtubule (closed symbols) and actin (open symbols) channels of the first ( $t_{i}$, squares) and last $\left(t_{f}\right.$, triangles) frames of three $\sim 6$-min videos (SI Movies S1-S3) representative of the three broad Classes (1-3) we observe across the composite formulation space. Class 1 (purple, top) has limited spatial correlations that decay over short ranges, Class 2 (orange, middle) exhibits broad mesoscale correlations with minimal decay, and Class 3 (magenta, bottom) displays the highest small-scale correlations that decay on length scales between that of Class 1 and 2. B. Structural correlation lengths $\xi$, determined from fits of each $g(r)$ curve (172 in total) to $g(r)=g(0) e^{-r / \xi}$ versus time, for actin (open, A) and microtubules (closed, MT) in composites with no crosslinking (blue, top), actin crosslinking (green, middle) and microtubule crosslinking (red, bottom) and driven by kinesin ( $\mathrm{K}$, dark shades) or both kinesin and myosin $(\mathrm{K}+\mathrm{M}$, light shades), where $t=0$ is the time at which kinesin is added to the composites. Each data point is from a different video and is an average of the correlation lengths determined for the first and last frame of that video. The data points that are computed from the curves shown in A are circled in the corresponding color in B. In general, $\xi$ increases in time for both actin and microtubule networks in all composites systems, and composites driven solely by kinesin have greater correlation lengths than those in which myosin is also present. 
By fitting each $g(r)$ curve (computed separately for actin and microtubules at 12-16 different time-points for each of the 6 formulations) to an exponential function $g(r)=g(0) e^{-r / \xi}$ we determine a corresponding characteristic network correlation length $\xi$ that is a measure of the average feature size. As shown in Figure 2B, $\xi$ increases substantially over time for all composites, with the largest increase for composites without crosslinkers (Fig 2B, top panel) and smallest for microtubule-crosslinked composites (Fig 2B, bottom panel). This result corroborates the Fig 1C images which show increased heterogeneity and clustering over time that is strongest without crosslinking and weakest with MT crosslinking. Further, the presence of myosin generally results in smaller $\xi$ values compared to kinesin-only composites, with this difference being largest without crosslinkers and smallest with microtubule crosslinking, in line with the more connected meshlike networks that myosin confers compared to more clustering and separation without myosin.

Notably, without crosslinkers, the MT correlation length, $\xi_{M T}$, increases monotonically over $\sim 20$ fold, while the actin network correlation length, $\xi_{A}$, exhibits a smaller non-monotonic increase. This result corroborates the qualitative description of MTs progressively moving from an interconnected mesh (small $\xi_{M T}$ ) to a distribution of amorphous clusters that grow in size over time (increasing $\xi_{M T}$ ) and initially take up actin filaments (increasing $\xi_{A}$ ) after which actin filaments are squeezed out into the surrounding environment and form a connected mesh (reducing $\xi_{A}$ ). Actin crosslinking prevents this decoupling by allowing for sustained interpenetration of actin and microtubule networks.

Different Classes exhibit distinct directionality, spatial heterogeneity, and time-evolution of flow fields. This complex reconfiguration phase space (Figs 1,2) motivated us to investigate how the actin and microtubules in the composites move from state to state over time. We first evaluate the actin and microtubule velocity flow fields by performing particle image velocimetry (PIV) on the example Class 1, 2 and 3 videos we analyzed in Fig 2A (Fig 3A). The vector arrows show the velocity direction and absolute magnitude while the underlying grayscale map depicts the normalized speed distribution. Class 1 flow fields for both actin and microtubules show no preferred direction, quantified by the broad histogram of angular orientations (Fig 3B, top panel), and minimal spatial correlation of magnitudes, depicted by the spatially heterogeneous pattern of the grayscale map (Fig 3A, top panel).

Class 2 PIV fields show starkly different dynamics with all velocity vectors pointing in nearly the same direction with spatially coordinated magnitudes (Fig 3A, Class 2). The standard deviations of orientations $\sigma_{i}$ and $\sigma_{f}$ are $\sim 40 \mathrm{x}$ smaller than those for Class 1 (Fig 3B), and the direction (i.e., the position of the distribution peak) and spread (i.e., $\sigma_{i}$ and $\sigma_{f}$ ) show negligible time dependence, as seen by the overlapping distributions (Fig 3B, Class 2).

Interestingly, the Class 3 system displays features of both Class 1 and Class 2 depending on the time. Initially, there is less spatial coordination of speeds, shown by the grayscale maps in the $t_{i}$ images (Fig 3A, Class 3), similar to Class 1 , albeit with a smaller $\sigma_{i}$ (Fig 3B, Class 3, $t_{i}$ ). However, at $t_{f}$ the direction of motion is nearly orthogonal to the starting direction with a tighter and more spatially coordinated speed distribution, similar to Class 2 (Fig 3B, Class $3, t_{f}$ ). 

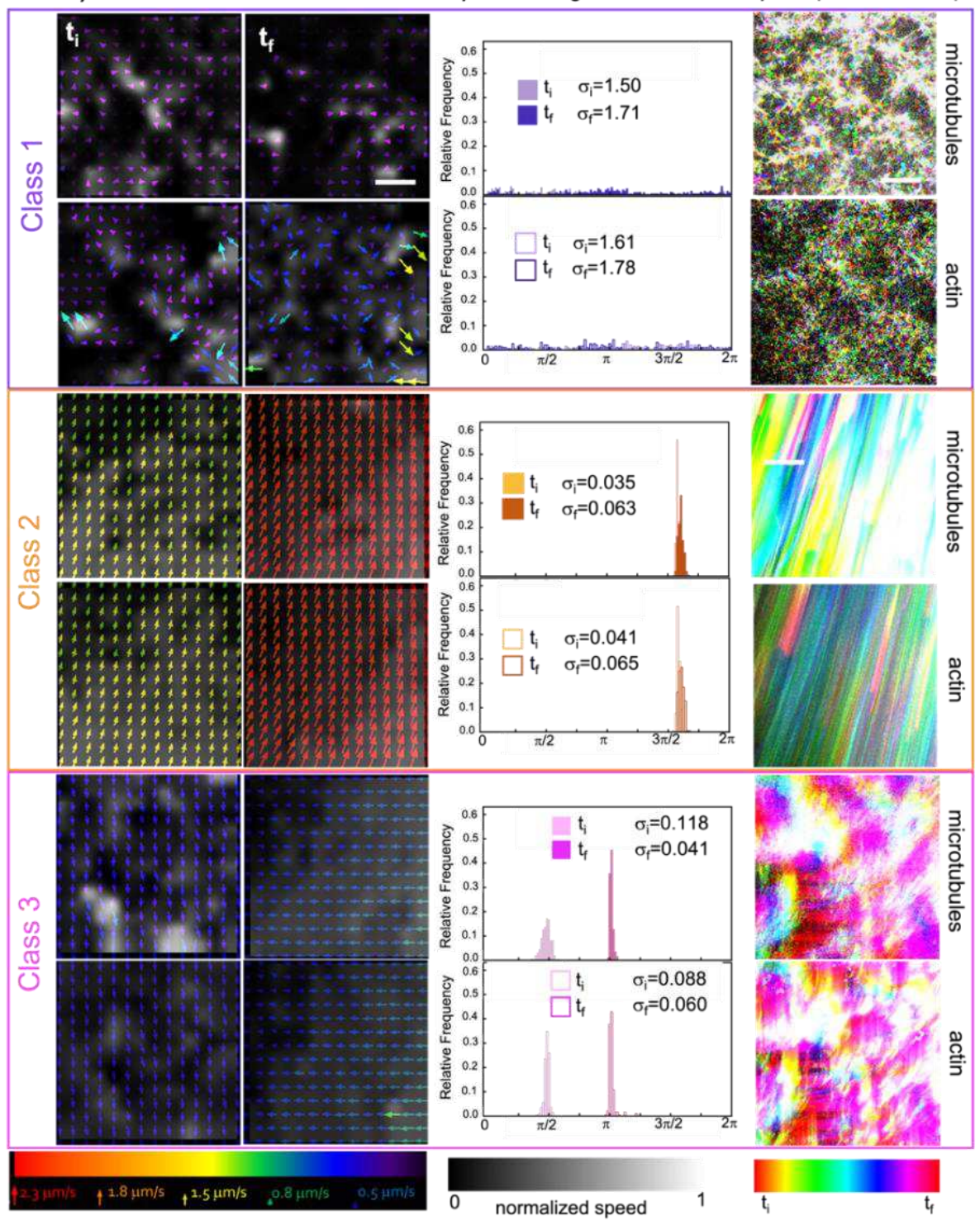

Figure 3. Different classes of active composites exhibit spatiotemporally-distinct flow fields. A. Particle Image Velocimetry (PIV) showing flow fields for the first $\left(t_{i}\right)$ and last $\left(t_{f}\right)$ frames of the videos analyzed in Figure 2 that represent Class 1 (top, purple), Class 2 (middle, orange), and Class 3 (bottom, magenta). Velocity flow fields for microtubule (top) and actin (bottom) channels are shown for each Class with arrow colors corresponding to the universal speed scale at the bottom, and the grayscale colormap showing the spatial distribution of speeds, normalized separately for each flow field (scale shown at bottom). Scale bar in top panel is $50 \mu \mathrm{m}$ and applies to all images. B. Distributions of angles (in radians) of velocity vectors shown in A with corresponding standard deviations $\sigma_{i}$ and $\sigma_{f}$ listed. C. Temporal color maps showing the frame-to-frame position of each pixel relative to its starting point for the videos analyzed in A and B. Class 1 maps shows small-scale random motion; Class 2 maps depict fast motion that is largely unidirectional across the entire field of view for the duration of the video; Class 3 maps reveal motion that has features of both Class 1 and 2. Temporal color maps for 3 more representative videos for each class are shown in SI Fig S1. 
For each movie, we create a temporal color map that shows the position of each pixel relative to its starting point in each color-coded subsequent frame (Fig 3C, SI Fig S1). Class 1 color maps show small-scale motion with uncorrelated directionality. Class 2 shows fast spatially coordinated motion that is nearly unidirectional over the course of the video. Class 3 color maps exhibit both Class 1 small-scale restructuring and Class 2 flow indicated by the swirling patterns and large regions of similar color.

Actin and microtubules exhibit coordinated ballistic motion with a distribution of speeds and multi-mode dynamics. To fully characterize the type and time-dependence of the motion shown in Figure 3 and its dependence on composite formulation, we perform differential dynamic microscopy (DDM) on the same 43 time-series that we analyze using SIA. As described in Methods, DDM analyzes differences of images separated by varying lag times $\Delta t$ in Fourier space to compute image structure functions $D(q, \Delta t)$ for different wavevectors $q$ which show how quickly density fluctuations become decorrelated for a given spatial scale (i.e., wave vector $q$ ) (Fig 4A) and the corresponding functional form of the decorrelation. Often, the image structure function $D(q, \Delta t)$ is fit to an exponential function to determine a characteristic decay time, $\tau$, of density fluctuations for each accessible length scale $2 \pi / q$. Figure $4 \mathrm{~A}$ shows sample $D(q, \Delta t)$ curves for a specific $q$ value for the actin and microtubule channels of the representative Class 1,2 , and 3 videos analyzed in Figure 3. While Class 1 curves show simple slow rise to plateau at large lag times (Fig 4A, top panel), Class 2 curves exhibit oscillations in the decorrelation plateau (Fig 4A, middle panel), and Class 3 curves reveal two distinct plateaus that occur at well-separated lag times (Fig 4A, bottom panel). These non-trivial functional forms cannot be accurately described by exponential functions typically used in $\mathrm{DDM}^{26,28,58,59}$, so we instead use a function that assumes Schulz distributions of velocities, as has been used in other ballistic biological systems such as swimming E. coli ${ }^{60,61}$ (see Methods). This function captures the Class 2 oscillatory plateaus for fast dynamics, and a sum of two Schulz velocity distributions accurately captures the two-plateau Class 3 behavior.

From the $D(q, \Delta t)$ fits, we extract the decay times, $\tau(q)$, which exhibit a power-law dependence on the wavevector $q$ used to further quantify the type and rate of motion (Fig 4B). Systems that undergo ballistic motion, as previously reported for myosin-driven cytoskeleton composites ${ }^{26,28}$, exhibit the scaling $\tau \sim(v q)^{-1}$ where $v$ is the average velocity. As shown, despite the varied functional forms of $D(q, \Delta t)$, all Classes exhibit ballistic scaling across all measured wave vectors (Fig 4B). Class 3 curves have two distinct $\tau(q)$ curves with corresponding speeds that differ by a factor of $\sim 4$. The 'fast' and 'slow' Class 3 speeds are comparable to those measured for the Class 2 and Class 1 examples, respectively, indicating that Class 3 composites undergo slow restructuring (Class 1) and coordinated flow (Class 2). We note that while Class 1 exhibits slow motion that is spatially decorrelated, the motion at any given point in the field of view is ballistic rather than diffusive, as previously reported for myosin-driven composites ${ }^{26}$. It is the directionality that is spatially decorrelated.

Corresponding kymographs, performed along the $x$ and $y$ directions of each video analyzed in Figure 4A,B, depict these multi-mode dynamics and their associated directionality (Fig 4C). The different colors indicate directionality $(+x /+y,-x /-y$, or no motion) and the steepness of the lines correlate with the speed. Class 1 composites display minimal motion that is similar in both directions, as shown by largely horizontal streaks of all colors. Class 2 composites exhibit clear directional dependence with rapid large-scale $+y$ motion (steep magenta streaks in the $y$-axis 
panels) while there is similar $+x$ and $-x$ motion (opposing shallow magenta and green streaks in the $x$-axis panels). Class 3 kymographs display features from both Class 1 and 2, with directed Class 3-type motion (steeper streaks) more apparent at later times, as also seen in PIV (Fig 3A).

In the following sections, we use the distinct $D(q, \Delta t)$ characteristics described above to correlate Class with composite formulation and time. Namely, Class 1 is defined by a $D(q, \Delta t)$ curve that exhibits a single steady high- $\Delta t$ plateau, Class 2 displays oscillations in the $D(q, \Delta t)$ plateau, and Class 3 has two distinct plateaus (Fig 4A).
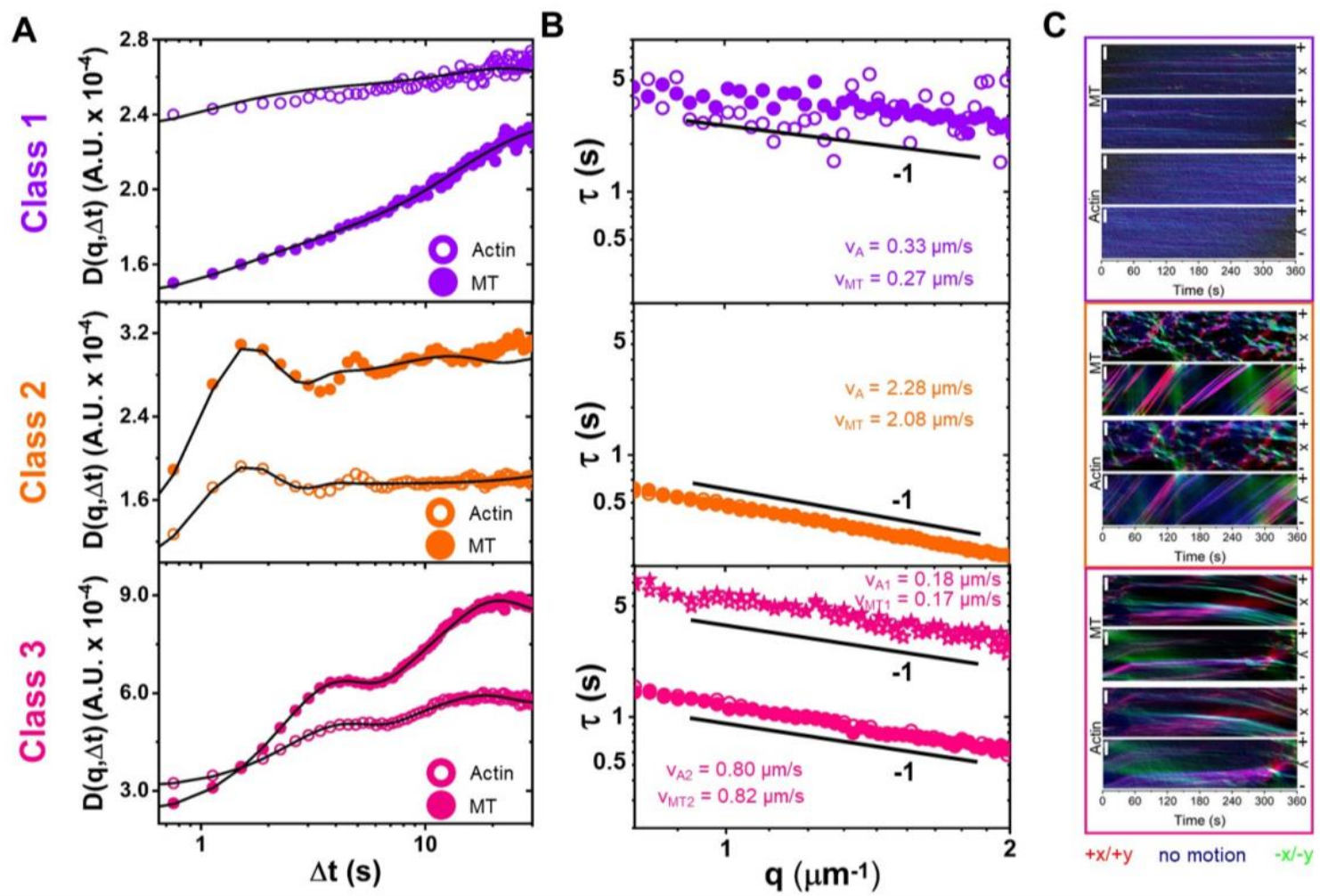

Figure 4. Differential dynamic microscopy and kymographs characterize the multi-mode ballistic dynamics the different Classes exhibit. A. Representative DDM image structure functions $D(q, \Delta t)$ versus lag time $\Delta t$ computed for microtubule (closed symbols) and actin (open symbols) channels analyzed in Fig $2 \mathrm{~A}$ and 3 at a wavevector $q=1.33 \mu \mathrm{m}^{-1}$ with corresponding fits to functions described in Methods. Class 1 curves (top) exhibit slow decay to a decorrelation plateau; Class 2 curves (middle) reach plateaus at much smaller $\Delta t$ with apparent oscillations; Class 3 curves (bottom) display two distinct correlation plateaus that occur at lag times in between those of Class 1 and 2. B. Corresponding decay times $\tau(q)$ computed from $D(q, \Delta t)$ fits, which universally follow $\tau(q)=(v q)^{-1}$ scaling, indicative of ballistic motion with speed $v$. Speeds for actin $\left(v_{A}\right)$ and microtubules $\left(v_{M T}\right)$ determined from each $\tau(q)$ fit are listed. C. MT and actin kymographs computed for a horizontal ( $x$, top) and vertical ( $y$, bottom) line spanning the field of view for the duration of each video. The scale bar shown is $50 \mu \mathrm{m}$ and corresponds to the $y$-axis while the $x$-axis shows time. Color coding (red, green, blue) corresponds to directionality $(+x /+y,-x /-y$, no $x / y$ motion) as indicated below the kymographs. Steeper slopes correspond to faster motion. 
Competition between kinesin and myosin delays the onset of kinesin-driven acceleratory dynamics and suppresses multi-mode behavior. Now that we have identified quantitative metrics to classify different Classes of network dynamics, we can use these metrics to determine how the dynamics depend on composite formulation and activity time. First, we use DDM to evaluate the actin and microtubule velocities determined from the corresponding $\tau(q)$ for each time-series (513 per formulation) for each of the six composite formulations. Figure 5A shows time-varying effects of crosslinking (different panels) and motors (dark vs light shades in each panel), with velocities spanning over three orders of magnitude. Interestingly, actin and microtubule velocities are well-correlated (Fig 5A, comparing open and closed symbols) across all composites and times, despite the varying degrees to which we observe them colocalize or de-mix (Fig 1D, 2). This results agrees with the data in Figures 3 and 4, as well as recent work on myosin-driven composites ${ }^{26,28}$.

We also find that actin and microtubules in all composites accelerate and reach a maximum velocity $v_{\max }$ (dashed circles in Fig 5A), after which the velocity decreases. By extracting the maximum velocity and the time at which $v_{\max }$ is reached, $t\left(v_{\max }\right)$, we observe distinct differences depending on the crosslinking scheme and the active motors employed (Fig 5B), which we describe below. By assigning each data point to a specific Class based on its $D(q, \Delta t)$ characteristics, we also correlate the measured speeds with Class (Fig 5D). As shown in Figure $5 \mathrm{C}$, the average Class 1 speed $\left(\bar{v}_{1} \simeq 0.15 \mu \mathrm{m} / \mathrm{s}\right)$ is an order of magnitude slower than that of Class $2\left(\bar{v}_{2} \simeq 1.8 \mu \mathrm{m} / \mathrm{s}\right)$ and the average fast and slow speeds for Class 3 are comparable to those of Class 1 and 2, respectively $\left(\bar{v}_{3, \text { slow }} \simeq 0.17 \mu \mathrm{m} / \mathrm{s}, \bar{v}_{3, \text { fast }} \simeq 1.7 \mu \mathrm{m} / \mathrm{s}\right)$.

For the uncrosslinked composite with kinesin motors (no myosin) the velocities of both actin and microtubules increase $\sim 20$-fold in the first $\sim 20 \mathrm{mins}$, from $v_{i} \simeq 0.3 \mu \mathrm{m} / \mathrm{s}$ to $v_{\max } \simeq 7 \mu \mathrm{m} / \mathrm{s}$ (Fig $5 \mathrm{~A}$, top panel). Further, a second dynamic mode with a slower speed $v_{\text {slow }}$ emerges at 12 mins (blue stars, Fig 5A), with a magnitude similar to $v_{i}$, while the corresponding fast speed at this time is close to $v_{\max }\left(v_{\text {fast }} \simeq 3 \mu \mathrm{m} / \mathrm{s}\right)$. Following this time-point the slow mode $\left(v_{\text {slow }}\right)$ is no longer detectable and $v_{\text {fast }}$ first increases to $v_{\max }$ then decreases. This result suggests that the initial motion (i.e., $\left.v_{i}\right)$ is due to slow restructuring $\left(v_{i} \simeq v_{\text {slow }}\right)$ reminiscent of Class 1 behavior, followed by a period of multi-mode Class 3 dynamics (i.e., both restructuring with $v_{\text {slow }}$ and flow with $\left.v_{\text {fast }}\right)$, after which Class 2 fast directed flow takes over $\left(v \simeq v_{\max }\right)$. Crosslinking of either actin or microtubules has similar effects on the magnitude and time-evolution of the speed of the composites. In both cases, $v_{\max }$ and $t\left(v_{\max }\right)$ are reduced, with MT crosslinking having a more pronounced effect than actin crosslinking (Fig 5B).

Further, in both crosslinking cases, both a fast and slow speed are present at the first time-point ( $t=8 \mathrm{~min}$ ), but the slow mode (red and green stars, Fig 5A) is no longer detectable after this point for MT crosslinking. This effect likely arises from the reduced degrees of freedom and enhanced connectivity that crosslinking provides, which facilitates coordinated mesoscale motion (Class 2 fast speeds) and suppresses uncorrelated microscale restructuring (Class 1 slow speeds). Microtubule crosslinking has a more apparent effect as it is the substrate on which kinesin acts.

Introducing myosin substantially delays the onset of acceleratory dynamics and increases $t\left(v_{\max }\right)$ for all composites, while $v_{\max }$ is similar with and without myosin (Fig 5A,B). Further, for the unlinked composites the presence of myosin leads to Class 3 multi-mode behavior occurring in the first two time-points (light blue stars, Fig 5A), but the corresponding $v_{\text {slow }}$ and $v_{\text {fast }}$ are both reduced by an order of magnitude. Moreover, the presence of crosslinkers eliminates this initial multi-mode behavior (no light green or light red stars in Fig 5A). Instead, only a single speed, 
comparable to $v_{\text {slow }}$ in the corresponding composites without myosin, is present. These findings suggest that myosin activity suppresses Class 2 flow, and crosslinking further arrests this motion.
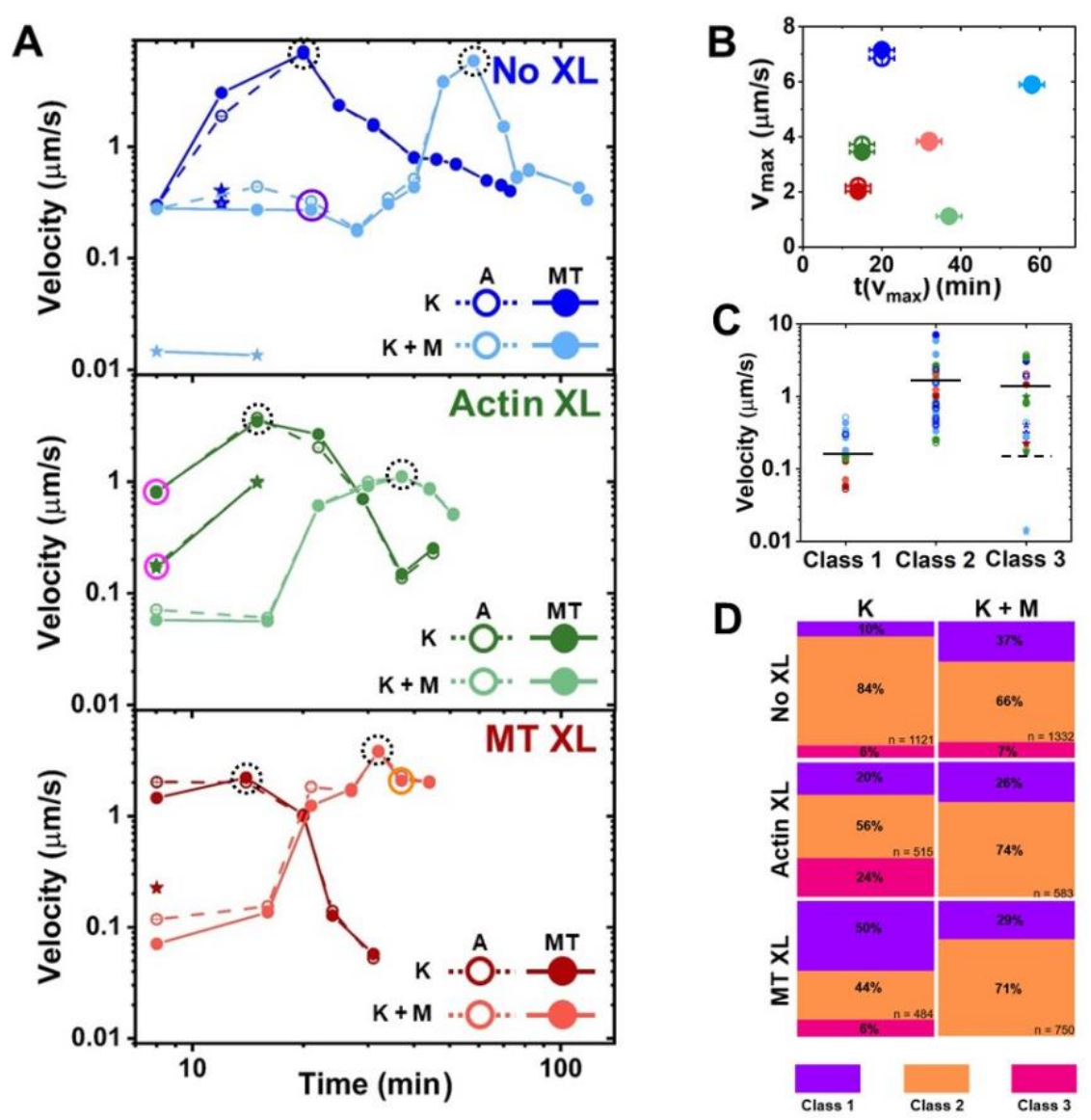

Figure 5. Velocities measured via DDM show acceleration and deceleration phases of composites, with distinct dynamic Classes, programmed by crosslinking and myosin activity. A. Velocities of microtubules (MT, closed circles) and actin (A, open circles) as a function of activity time in kinesin-driven composites with no crosslinking (top, blue), actin crosslinking (middle, green), and microtubule crosslinking (bottom, red); and without myosin (K, darker shades) and with myosin ( $\mathrm{K}+\mathrm{M}$, lighter shades). For Class 3 cases, which have two velocities, and only occur at early times for each formulation, the slower speed is indicated by a star. Data points corresponding to the $\tau(q)$ curves shown in Fig 4 are circled in the corresponding Class color. Data points enclosed by dashed black circles correspond to $v_{\max }$ for each formulation. Error bars (most too small to see) are the standard error over the power-law fits of the corresponding $\tau(q)$. B. Maximum velocity $v_{\max }$ reached by each composite plotted against the time at which $v_{\max }$ occurs. Error bars along the $x$-axis indicate the duration of the video over which the velocity is computed. C. Scatterplot of all 106 measured actin and MT velocities shown in A, divided into Class and color-coded as in A and B. Horizontal lines indicate averages across the data shown, the dashed horizontal line for indicates the average of the slower velocities (stars) in Class 3. D. Percentage of data for each composite formulation (as indicated on the left and top) that has Class 1 (purple), Class 2 (orange) or Class 3 (magenta) characteristics, as determined via the functional forms of $D(q, \Delta t)$. The number of $D(q, \Delta t)$ curves $n$ over which the percentages are computed are listed. In general, all $D(q, \Delta t)$ curves for actin and microtubules for a given video (5-13 per formulation) have the same Class characteristics. 
Moreover, these results indicate that the fast Class 2 motion is due to kinesin-driven motion (i.e., minimal change in $v_{\max }$ ), and that myosin activity counteracts kinesin activity to delay the acceleration to Class 2 dynamics, rather than cooperating synergistically to amplify the active dynamics. Such competition may arise due to the different stiffnesses of the substrates which leads to contractile stresses in actin filaments and extensile stresses in microtubules ${ }^{62}$. The differences in initial velocities for kinesin-only versus kinesin-myosin composites further highlight the accelerated activity for crosslinked versus unlinked composites: without crosslinkers, the initial velocities of both composites are nearly identical (initially overlapping light and dark blue circles in Fig 5A) and an order of magnitude slower than $v_{\text {max }}$, whereas for both crosslinked cases, the initial velocity is an order of magnitude higher (close to $v_{\max }$ ) than that without myosin (i.e. the first dark green and red circles are higher than corresponding light shades in Fig 5A).

To quantify the propensity for different formulations to exhibit different Classes of dynamics, we evaluate the percentage of data for each formulation that fall into each Class (Fig 5D). We find that for kinesin-only composites, crosslinking increases the percentage of Class 1 and 3 dynamics and reduces fast Class 2 dynamics. Because the maximum speeds reached for crosslinked composites are lower than that for the unlinked composite (Fig 5B) there are fewer time-points at which the speeds are fast enough to elicit Class 2 type behavior (i.e., oscillations in $D(q, \Delta t)$ plateau). The addition of myosin to the unlinked composite suppresses fast Class 2 flow and increases the fraction of videos that undergo slow restructuring (Class 1 and 3), demonstrating the competition between kinesin and myosin activity that serves to hinder Class 2 flow. Conversely, the addition of myosin to crosslinked networks eliminates Class 3 dynamics and increasing the propensity to undergo fast flow. This surprising result likely arises from the reduced degrees of freedom of crosslinked networks, as described above, which limits the extent to which motors can induce uncorrelated microscale restructuring, instead facilitating coordinated flow.

The structure-dynamics phase space of motor-driven composites can be described by the varying degrees to which different Classes store or dissipate motor-generated forces. To determine the mechanisms underlying the complex restructuring and dynamics presented in Figs 2-5, and map the phase space of possible structure-dynamics relationships, we correlate DDM-measured velocities $v$ to SIA-measured correlation lengths $\xi$ for all formulations and times (Fig 6).

To shed light on the restructuring that occurs during the acceleratory phase, we consider $\xi_{A}$ and $\xi_{M T}$ at the time-point when $v_{\max }$ is reached (Fig 6A). For all composites, the correlation length for the actin network is larger than that for the microtubules when the composite is moving fastest (i.e., $\xi_{A}>\xi_{M T}$ at $v_{\max }$ ), and myosin activity increases this separation in length scales and increases $\xi_{A}$. This result implies that the actomyosin activity contracts the actin network independently of the microtubules, rather than allowing it to simply move along with the kinesin-driven microtubule network.

Next, we examine the velocity of the networks at the point when actin and microtubules display their respective maximum correlation lengths $\xi_{\max , A}$ and $\xi_{\max , M T}$, which correspond to the most heterogeneous and/or clustered structures (Fig 6B). We note that $\xi_{\text {max }}$ is greater for MTs than for actin in kinesin-driven systems, as we may expect since kinesin acts on microtubules. When myosin is present, we find the opposite (i.e., $\xi_{\max , A}>\xi_{\max , M T}$ ), suggesting that myosin can more easily restructure actin compared to kinesin acting on microtubules, likely due to the comparative flexibility of actin. While the corresponding velocities differ by an order of magnitude without 
crosslinking, the speeds for actin and MTs are similar when either type of crosslinking is present, suggestive of more connectivity and coordination as reflected in Fig 1C,D.
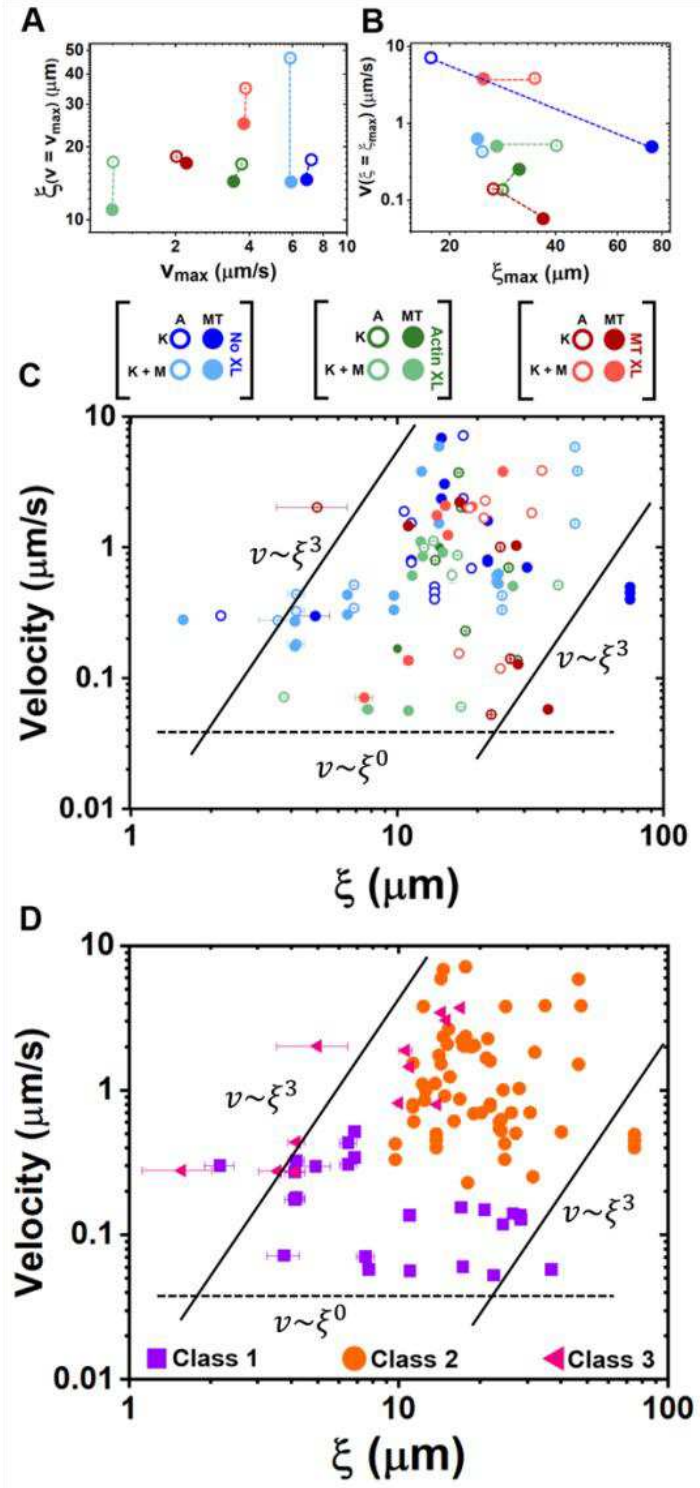

Figure 6. Phase space of structure-dynamics relationships in motor-driven cytoskeleton composites. A. Maximum velocity $v_{\max }$ for actin (open symbols) and MTs (closed symbols) for all composite formulations versus the correlation length $\xi$ at the time at which the corresponding $v_{\max }$ occurs (colorcoded according to legend shown below the plot). Dashed lines connect data for MTs and actin determined for the same composite formulation. B. Maximum correlation length $\xi_{\max }$ measured for actin (open symbols) and MTs (closed symbols) for all composite formulations versus the corresponding velocity $v$ at the time at which $\xi_{\max }$ occurs (color-coded according to the legend as in A). Dashed lines connect data for MTs and actin determined for the same formulation. C. Relationship between velocity and correlation length for all composite formulations and time-points for both MTs and actin (color-coded according to the legend as in A and B). Scaling lines show power-law scaling corresponding to $v \sim \xi^{3}$ and $v \sim \xi^{0}$ expected for motor-driven elastic and dissipative systems (as described in the text). D. Same as $\mathbf{C}$ but colors and symbols correspond to Class as listed in the legend. 
Finally, we evaluate the relationship between $v$ and $\xi$ for all composites over all time-points (Fig 6C,D), to demonstrate the range of possible dynamics and structural properties that can be programmed with our platform and elucidate underlying structure-dynamics interactions. Considering an active elastic network, the motors generate forces in the network which result in an acceleration $a$. The velocity in this case is $v \sim a \tau$, where $\tau$ is the relaxation time between successive contacts (i.e., crosslinks or entanglements) within the network, which scales inversely with the elastic plateau modulus $G$ (i.e., $\left.\tau \sim G^{-1}\right)^{63}$. We also recall that the plateau modulus $G$ for a network of semiflexible filaments scales as $G \sim \ell^{-3}$ where $\ell$ is the mesh size ${ }^{64}$, which scales as the correlation length $\xi$. Combining these relations yields $v \sim \xi^{3}$. Indeed, the distribution of data points shown in Figure 6C fills a region of the phase space enveloped by $v \sim \ell^{3}$ scaling. Colorcoding the data by Class (Fig 6D) reveals that this scaling is most apparent for Class 2 cases which exhibit fast directed motion and mesoscale structural correlations.

The low densities of static crosslinkers in our composites introduce a dissipative viscous component that should be more apparent in the composites without crosslinkers. For a viscousdominated system motor-generated forces should result in a drift velocity $v$ rather than an acceleration $a$, and thus should have minimal dependence on $\xi$. This effect is evident for the lower velocities in which the scaling deviates from $v \sim \xi^{3}$ and instead more closely follows $v \sim \xi^{0}$. This scaling is clear for the composites without static crosslinkers (Fig 6C) and those grouped as Class 1 (Fig 6D). The closer agreement with $v \sim \xi^{0}$ versus $v \sim \xi^{3}$ at lower velocities (Class 1 purple data points) compared to fast speeds (Class 2 orange data), which more closely follow $v \sim \xi^{3}$ scaling, can be understood as arising from the terminal behavior of viscoelastic networks ${ }^{64}$. At lower strain rates, the network has more time to relax imposed stress and thus exhibit more fluidlike rather than elastic-like dynamics. Class 1 cases, which demonstrate microscale rearrangement and random, decorrelated motion, are best described by $v \sim \xi^{0}$ scaling (Fig 6D), suggesting that the filaments have time to bend and reconfigure on the timescale of the force-generation, allowing for restructuring rather than the coordinated directed motion of Class 2.

\section{Conclusion}

The cytoskeleton is a non-equilibrium multifunctional composite composed of diverse protein filaments, force-generating motors, and crosslinkers that cooperate and compete to enable diverse cellular structures and processes. As such, the cytoskeleton serves as one of the primary inspirations to the burgeoning field of active matter, and much of current active matter research seeks to learn from and emulate the cytoskeleton. The composite nature of the cytoskeleton, which confers its signature versatility and programmability, is one of its hallmarks; yet, current active matter platforms are largely limited to a single force generating component and/or substrate.

Here, we address this gap by engineering co-entangled and crosslinked composites of microtubules and actin filaments driven by kinesin and myosin motors - breaking new ground in active matter design by incorporating multiple independently tunable force-generating components and active substrates.

By coupling structural and dynamical Fourier analyses (DDM and SIA) we show that composites undergo a combination of fast advective flow, slow ballistic restructuring, and multi-mode dynamics that result in structures ranging from interpenetrating actin-microtubule scaffolds to demixed amorphous clusters of separate filament types. Surprisingly, competition between kinesin 
and myosin force-generation delays the onset of restructuring and slows active motion while, at the same time, sustaining mixed filamentous networks of actin and microtubules. Conversely, crosslinking of actin or microtubules accelerates the time-evolution of active dynamics by enhancing cooperative actin-microtubule dynamics and suppressing microscale phase separation. Importantly, the emergent dynamics and extensive programmable phase space of non-equilibrium properties we reveal are a result of very subtle changes in substrate connectivity and activity. Finally, we discover that this rich phase space can be broadly divided into three Classes with distinct structure-dynamics relationships that we suggest arise from the varying degrees to which the Classes store or dissipate motor-generated forces.

Our work not only brings reconstituted cytoskeleton composites one important step closer to mimicking the complexity of the cell cytoskeleton in processes such as wound healing, mitosis,

and cytoplasmic streaming 7,15,16,43,65,66, but also opens the door for responsive reconfigurable materials that can be programmed to undergo multiple types of motion and reorganization over different spatiotemporal scales, with each component independently tunable across a broad parameter space. We expect our platform and results to spark new theoretical investigations into composite active matter and new experiments that explore the broad parameter space of this platform.

\section{Methods}

Protein Preparation: Rabbit skeletal actin monomers (Cytoskeleton, AKL99, Lot\#139), biotinactin monomers (Cytoskeleton, AB07, Lot\#49), porcine brain tubulin dimers (Cytoskeleton, T240, Lot\#121), biotin-tubulin dimers (Cytoskeleton, T333P, Lot\#27), rhodamine-labeled tubulin dimers (Cytoskeleton, TL590M, Lot\#31), and myosin-II (Cytoskeleton, MY02, Lot\#19), are reconstituted and flash-frozen into single-use aliquots according to previously described protocols ${ }^{28,48}$.

Biotinylated kinesin-401 is expressed in Rosetta (DE3)pLysS competent E. coli (ThermoFisher) and grown on selective media plates for $16-18$ hours at $37^{\circ} \mathrm{C}$. Fifteen colonies are added to a $5 \mathrm{ml}$ starter culture of selective LB media and grown for 2 hours at $37^{\circ} \mathrm{C} / 250 \mathrm{rpm}$ before adding to 400 $\mathrm{ml}$ of selective LB media. Cells are grown at $37^{\circ} \mathrm{C} / 250 \mathrm{rpm}$ to OD 0.6-0.9 at $600 \mathrm{~nm}$, then induced at $20^{\circ} \mathrm{C} / 250 \mathrm{rpm}$ for 18 hours with $1 \mathrm{mM}$ Isopropyl $\beta$-D-1-thiogalactopyranoside (IPTG), and pelleted at 5,000 rpm for 10 minutes at $4^{\circ} \mathrm{C}$ before being frozen at $-80^{\circ} \mathrm{C}$ for 1 hour. Cells are lysed in lysis binding buffer $\left(50 \mathrm{mM}\right.$ PIPES, $4 \mathrm{mM} \mathrm{MgCl}_{2}, 20 \mathrm{mM}$ imidazole, $10 \mathrm{mM}$ mercaptoethanol, $50 \mathrm{uM}$ ATP, one protease inhibitor tablet per $10 \mathrm{ml}, 1.1 \mathrm{mg} / \mathrm{ml} \mathrm{PMSF}, 1.1 \mathrm{mg} / \mathrm{ml}$ lysozyme) via sonication for 3 mins, pulsing every 20 seconds, then pelleted for 30 mins at 40,000 $\mathrm{x} \mathrm{g}$ at $4^{\circ} \mathrm{C}$, filtered through a $0.22 \mathrm{uM}$ filter, and incubated with $1 \mathrm{ml}$ nickel (Ni-NTA) agarose beads (Qiagen) for 2 hours on a rocker at $4{ }^{\circ} \mathrm{C}$. The lysate/bead mixture is passed through a chromatography column then washed with $15 \mathrm{ml}$ buffer (50mM PIPES, 4mM MgCl2, 20mM imidazole, $10 \mathrm{mM} \beta$-mercaptoethanol, 50uM ATP, one protease inhibitor tablet per $10 \mathrm{ml}$ ) before $1000 \mu \mathrm{l}$ fractions are eluted in $(50 \mathrm{mM}$ PIPES, $4 \mathrm{mM} \mathrm{MgCl} 2,20 \mathrm{mM}$ imidazole, $10 \mathrm{mM} \beta$ mercaptoethanol, 50uM ATP, one protease inhibitor tablet per 10ml, 2mM DTT, 0.05mM ATP). An elution dot blot is performed to assess the most concentrated fraction which is run through a 40K MWCO desalting column for buffer exchange with PEM100 with $0.1 \mathrm{mM}$ ATP, then mixed with $60 \%$ sucrose for a final concentration of $10 \%$ sucrose before being aliquoted and flash-frozen into single-use aliquots. 
For composites that incorporate actin or microtubule crosslinking, actin-actin or microtubulemicrotubule crosslinker complexes are prepared according to previously described protocols ${ }^{48}$. In brief, biotin-actin or biotin-tubulin is combined with NeutrAvidin and free biotin at a ratio of 2:2:1 protein:free biotin:NeutrAvidin.

Immediately prior to experiments: (1) myosin-II is purified as previously described ${ }^{27}$ and stored at $4^{\circ} \mathrm{C}$, and (2) kinesin clusters are formed by incubating the dimers at a 2:1 ratio with NeutrAvidin (ThermoFisher) with $125 \mu \mathrm{M}$ DTT for 30 minutes at $4^{\circ} \mathrm{C}$.

Active Cytoskeleton Composite Preparation: Actin-microtubule composites are formed by polymerizing $2.32 \mu \mathrm{M}$ unlabeled actin monomers and $3.48 \mu \mathrm{M}$ tubulin dimers $(5 \%$ rhodaminelabeled) in PEM-100 (100 mM PIPES, $2 \mathrm{mM} \mathrm{MgCl}_{2}$, $2 \mathrm{mM}$ EGTA) supplemented with 0.1\% Tween, $10 \mathrm{mM}$ ATP, $4 \mathrm{mM}$ GTP, $5 \mu \mathrm{M}$ Taxol, and $0.47 \mu \mathrm{M}$ AlexaFluor488-phalloidin (Life Technologies, A12379) to label the actin.

For crosslinked composites, a portion of either the actin monomers or the tubulin dimers is replaced with equivalent crosslinker complexes to achieve the same overall actin and tubulin concentrations and crosslinker:protein ratios of $R_{A}=0.02$ for actin or $R_{M T}=0.005$ for microtubules. $R_{A}$ and $R_{M T}$ values are chosen to achieve similar lengths between crosslinkers $d$ along actin filaments and microtubules $\left(d_{A} \simeq 60 \mathrm{~nm}\right.$ and $\left.d_{M T} \simeq 67 \mathrm{~nm}\right)$. As previously described ${ }^{48}$, we estimate these values using $d_{A}=\frac{l_{\text {monomer }}}{2 R}$, where $l_{\text {monomer }}$ is the length of an actin monomer, and $d_{M T}=\frac{l_{\text {ring }}}{26 R}$, where $l_{\text {ring }}$ is the length of a ring of 13 tubulin dimers. Crosslinking ratios are also tuned to be high enough to induce measurable changes in the viscoelastic properties compared to unlinked networks, but low enough to prevent filament bundling ${ }^{47}$.

Actin and tubulin concentrations are chosen to be similar to those used in previous studies on myosin-driven actin-microtubule composites ${ }^{28}$, and such that the mesh sizes for the actin and microtubule networks are comparable $\left(\zeta_{A} \simeq 0.96 \mu \mathrm{m} \text { and } \zeta_{M T} \simeq 1.44 \mu \mathrm{m} \text {, respectively }\right)^{63}$. Further fine-tuning of the concentrations is achieved through a series of optimization experiments to identify a formulation space in which composites reliably form percolated networks and are visibly active on the timescale of minutes.

Composites are polymerized for 30 mins at $37^{\circ} \mathrm{C}$, after which $1.86 \mu \mathrm{M}$ unlabeled phalloidin is added and the composite is incubated for $10 \mathrm{mins}$ at room temperature. $50 \mu \mathrm{M}$ blebbistatin is added to inhibit myosin-actin interaction prior to de-activation via $488 \mathrm{~nm}$ illumination ${ }^{26}$, and an oxygen scavenging system ( $45 \mu \mathrm{g} / \mathrm{mL}$ glucose, $0.005 \% \beta$-mercaptoethanol, $43 \mu \mathrm{g} / \mathrm{mL}$ glucose oxidase, 7 $\mu \mathrm{g} / \mathrm{mL}$ catalase) is added to reduce photobleaching. Finally, $0.47 \mu \mathrm{M}$ myosin-II and $0.35 \mu \mathrm{M}$ kinesin (pre-formed into complexes) are added. While myosin activity is controlled by blebbistatin de-activation, kinesin starts to act on microtubules immediately, so $t=0$ of each experiment is set as the time kinesin is added. Each sample is gently flowed into a sample chamber composed of a silanized ${ }^{67}$ coverslip and microscope slide fused together by a $\sim 100 \mu \mathrm{m}$ thick parafilm spacer and sealed with epoxy.

Fluorescence Microscopy: Imaging of AlexaFluor488-labeled actin and rhodamine-labeled microtubules comprising composites is performed using a Nikon A1R laser scanning confocal microscope with a 60x 1.4 NA oil-immersion objective (Nikon), a $488 \mathrm{~nm}$ laser with 488/525 nm excitation/emission filters, and a $561 \mathrm{~nm}$ laser with 565/591 nm excitation/emission filters. 488 $\mathrm{nm}$ illumination also locally activates myosin-II ATPase activity by de-activating blebbistatin as previously described ${ }^{26-28}$. Time-series (videos) of 256 x 256 pixel $(213 \mu \mathrm{m} \times 213 \mu \mathrm{m})$ images are 
collected at $2.65 \mathrm{fps}$ for a total of 1000 frames ( $6.28 \mathrm{mins}$ ). Imaging begins 5 mins after the addition of kinesin motors ( $t=5 \mathrm{~min}$ ) in the middle of the $\sim 100 \mu \mathrm{m}$ thick sample chamber. Each successive video) is collected in a different field of view of the same sample until there is no longer any discernible restructuring or motion ( 60 mins). 5-13 videos are collected for each of the six composite formulations (no crosslinking, actin crosslinking and microtubule crosslinking; with kinesin and with kinesin and myosin).

Differential Dynamic Microscopy (DDM): DDM is performed separately on the actin and microtubule channels of each 1000-frame video using custom written python scripts as described previously ${ }^{28}$. Radially-averaged image structure functions, $D(q, \Delta t)$ for each sampled wave vector $q$ are computed by taking fast 2D Fourier transforms of differences between images in the timeseries separated by a lag time $\Delta t . D(q, \Delta t)$ versus $\Delta t$ for each wave vector $q$ is fit to the sum of either one or two Schulz velocity distributions:

$A A\left(1-\left(\left[f\left(\frac{\tau_{1}\left(Z_{1}+1\right)}{Z_{1} * \Delta t} * \frac{\sin \left(Z_{1} * \arctan \left(\theta_{1}\right)\right)}{\left(1+\theta_{1}^{2}\right)^{\frac{Z_{1}}{2}}}\right)\right]+\left[(1-f)\left(\frac{\tau_{2}\left(Z_{2}+1\right)}{Z_{2} * \Delta t} * \frac{\sin \left(Z_{2} * \arctan \left(\theta_{2}\right)\right)}{\left(1+\theta_{2}^{2}\right)^{\frac{Z_{2}}{2}}}\right)\right]\right)\right)+B$,

where amplitude $A$, background $B$, decay times $\tau_{1}$ and $\tau_{2}$, amplitude fraction $f$, and Schulz numbers $Z_{1}$ and $Z_{2}$ are $q$-dependent free parameters, and $\theta_{n}=\frac{\Delta t}{\tau_{n}\left(Z_{n}+1\right)} 60$. Schulz numbers characterize the velocity distributions $P(v)=\frac{v^{z}}{Z !}\left(\frac{Z+1}{\bar{v}}\right)^{Z+1} \exp \left[-\frac{v(Z+1)}{\bar{v}}\right]$ where $Z=\left(\frac{\bar{v}}{\sigma}\right)^{2}-1$. Class 1 and 2 image structure functions exhibit a single decorrelation plateau and can be well-fit to a single Schulz distribution (i.e. $f=1$ ), while Class 3 curves exhibit two plateaus and are best fit to the sum of two modes with comparable $f$ values. $\tau(q)$ curves for each composite and time-point are extracted from the corresponding fits of $D(q, \Delta t)$. All composites exhibit $\tau(q) \sim q^{-1}$ scaling indicative of ballistic motion ${ }^{58}$ and the average velocity $v$ is computed by fitting $\tau(q)$ to $\tau(q)=$ $(v q)^{-1}$. Error bars shown in Figs 5 and 6 are standard error over each power-law fit, which includes 30-70 $q$ values over the range of $0.30 \mu \mathrm{m}^{-1} \leq q \leq 2.93 \mu \mathrm{m}^{-1}$.

Spatial Image Autocorrelation (SIA): SIA analysis is performed on the initial and final frames of the actin and microtubule channels of each 1000-frame time-series using custom Python scripts ${ }^{68}$. SIA measures the correlation in intensity $g$ of two pixels in an image as a function of separation distance $r^{69}$. Autocorrelation curves $g(r)$ are generated by taking the fast Fourier transform of the image $F(I)$, multiplying by its complex conjugate, and then applying an inverse Fourier transform $F^{-1}$ and normalizing by the squared intensity: $g(r)=\frac{F^{-1}\left(|F(I(r))|^{2}\right)}{[I(r)]^{2}}$. Structural correlation lengths $\xi$ are determined by fitting the decaying section of each autocorrelation curve $g(r)$ to an exponential $g(r)=g(0) e^{\frac{-r}{\xi}}$. Correlation lengths for actin and microtubules $\left(\xi_{A}\right.$ and $\left.\xi_{M T}\right)$ for each video, plotted in Figs 2 and 6 , are each an average of the values determined from the initial and final $g(r)$ curves for each video.

Dynamic Image Analysis: Particle Image Velocimetry (PIV) is performed on the first and last 20frame ( $7.5 \mathrm{~s}$ ) segment of each time-series using the PIV plugin for FIJI/ImageJ ${ }^{70}$. PIV produces a 64 x 64 vector flow field with each vector arrow representing the magnitude (color) and direction (angle) of the velocity at that point (Fig 3). Grayscale maps overlaying the vector maps show the spatial distribution of speeds normalized by the maximum speed, where white is the maximum and black represents no motion. Temporal color maps of all frames of each 1000-frame video are 
generated using the Temporal-Color Code FIJI/ImageJ plugin (Fig 3). Kymographs are created using the KymographClear FIJI/ImageJ plugin. A horizontal $(x)$ and a vertical $(y)$ line spanning the field of view of the first frame of each video is chosen for analysis, then the kymograph is generated by Fourier-filtering particles moving in different directions across the chosen path. Color coding shown in Fig 4 indicates forward $(+x /+y)$ motion, backward $(-x /-y)$ motion, and no motion.

Author Contributions: RMRA conceived the project, guided the experiments, interpreted the data, and wrote the manuscript. DHA performed the experiments, analyzed and interpreted the data, and wrote the manuscript. KAL purified and assayed the kinesin. JLR helped conceive the project, guide experiments, interpret data, and write the manuscript. MD helped interpret data, develop scaling arguments, and write the manuscript. CC performed experiments and analyzed data. JM, MH, and NS analyzed data and prepared figures. GL helped design experimental protocols and prepare reagents. RJM analyzed and interpreted data and provided useful feedback. JYS helped guide and perform experiments and analyze data. MJR helped guide experiments and provide useful feedback.

Competing Interest Statement: The authors declare no competing interests.

Acknowledgments: We thank Leila Farhadi for developing purification and handling protocols for kinesin clusters. This research was funded by a William M. Keck Foundation Research Grant and DMREF Award (DMR 2119663) awarded to RMRA., JLR., MD, and MJR; and a National Institutes of Health R15 Award (National Institute of General Medical Sciences award no. R15GM123420 awarded to RMRA and RJM.

\section{References}

1. Wen, Q. \& Janmey, P. A. Polymer physics of the cytoskeleton. Curr. Opin. Solid State Mater. Sci. 15, 177-182 (2011).

2. Xiao, Q., Hu, X., Wei, Z. \& Tam, K. Y. Cytoskeleton Molecular Motors: Structures and Their Functions in Neuron. Int. J. Biol. Sci. 12, 1083-1092 (2016).

3. Gardel, M. L., Schneider, I. C., Aratyn-Schaus, Y. \& Waterman, C. M. Mechanical integration of actin and adhesion dynamics in cell migration. Annu. Rev. Cell Dev. Biol. 26, 315-333 (2010).

4. Fletcher, D. A. \& Mullins, R. D. Cell mechanics and the cytoskeleton. Nature 463, 485-492 (2010).

5. Ajeti, V. et al. Wound healing coordinates actin architectures to regulate mechanical work. Nat. Phys. 15, 696-705 (2019).

6. Jung, W. et al. Dynamic motions of molecular motors in the actin cytoskeleton. Cytoskeleton 76, 517531 (2019).

7. Burla, F., Mulla, Y., Vos, B. E., Aufderhorst-Roberts, A. \& Koenderink, G. H. From mechanical resilience to active material properties in biopolymer networks. Nat. Rev. Phys. 1, 249-263 (2019). 
8. Pollard, T. D. \& O’Shaughnessy, B. Molecular Mechanism of Cytokinesis. Annu. Rev. Biochem. 88, 661-689 (2019).

9. Dogterom, M. \& Koenderink, G. H. Actin-microtubule crosstalk in cell biology. Nat. Rev. Mol. Cell Biol. 20, 38-54 (2019).

10. Huber, F., Boire, A., López, M. P. \& Koenderink, G. H. Cytoskeletal crosstalk: when three different personalities team up. Curr. Opin. Cell Biol. 32, 39-47 (2015).

11. Rivero, F. et al. The role of the cortical cytoskeleton: F-actin crosslinking proteins protect against osmotic stress, ensure cell size, cell shape and motility, and contribute to phagocytosis and development. J. Cell Sci. 109 ( Pt 11), 2679-2691 (1996).

12. Fürthauer, S., Needleman, D. J. \& Shelley, M. J. A design framework for actively crosslinked filament networks. New J. Phys. 23, 013012 (2021).

13. Kozlowski, C., Srayko, M. \& Nedelec, F. Cortical Microtubule Contacts Position the Spindle in C. elegans Embryos. Cell 129, 499-510 (2007).

14. Sun, B., Duclos, G. \& Stone, H. A. Network Characteristics of Collective Chemosensing. Phys. Rev. Lett. 110, 158103 (2013).

15. Buschmann, H., Green, P., Sambade, A., Doonan, J. H. \& Lloyd, C. W. Cytoskeletal dynamics in interphase, mitosis and cytokinesis analysed through Agrobacterium-mediated transient transformation of tobacco BY-2 cells. New Phytol. 190, 258-267 (2011).

16. Li, S., Sun, T. \& Ren, H. The functions of the cytoskeleton and associated proteins during mitosis and cytokinesis in plant cells. Front. Plant Sci. 6, 282 (2015).

17. Oakes, P. W. et al. Lamellipodium is a myosin-independent mechanosensor. Proc. Natl. Acad. Sci. U. S. A. 115, 2646-2651 (2018).

18. Das, A. et al. Stratification relieves constraints from steric hindrance in the generation of compact actomyosin asters at the membrane cortex. Sci. Adv. 6, eaay6093.

19. Banerjee, S., Gardel, M. L. \& Schwarz, U. S. The Actin Cytoskeleton as an Active Adaptive Material. Annu. Rev. Condens. Matter Phys. 11, 421-439 (2020).

20. Ross, T. D. et al. Controlling organization and forces in active matter through optically defined boundaries. Nature 572, 224-229 (2019).

21. Yadav, V. et al. Filament Nucleation Tunes Mechanical Memory in Active Polymer Networks. Adv. Funct. Mater. 29, 1905243 (2019).

22. Murrell, M. P. \& Gardel, M. L. F-actin buckling coordinates contractility and severing in a biomimetic actomyosin cortex. Proc. Natl. Acad. Sci. U. S. A. 109, 20820-20825 (2012).

23. Needleman, D. \& Dogic, Z. Active matter at the interface between materials science and cell biology. Nat. Rev. Mater. 2, 1-14 (2017).

24. Duclos, G. et al. Topological structure and dynamics of three-dimensional active nematics. Science 367, 1120-1124 (2020). 
25. Gagnon, D. A. et al. Shear-Induced Gelation of Self-Yielding Active Networks. Phys. Rev. Lett. 125, 178003 (2020).

26. Lee, G. et al. Myosin-driven actin-microtubule networks exhibit self-organized contractile dynamics. Sci. Adv. 7, eabe4334.

27. Sheung, J. et al. Motor-Driven Restructuring of Cytoskeleton Composites Leads to Tunable TimeVarying Elasticity. ACS Macro Lett. (2021).

28. Lee, G. et al. Active cytoskeletal composites display emergent tunable contractility and restructuring. Soft Matter 17, 10765-10776 (2021).

29. Berezney, J., Goode, B. L., Fraden, S. \& Dogic, Z. Extensile to contractile transition in active microtubule-actin composites generates layered asters with programmable lifetimes. ArXiv211000166 Cond-Mat Physicsphysics (2021).

30. Fürthauer, S. et al. Self-straining of actively crosslinked microtubule networks. Nat. Phys. 15, 12951300 (2019).

31. Foster, P. J., Fürthauer, S., Shelley, M. J. \& Needleman, D. J. Active contraction of microtubule networks. eLife 4, e10837 (2015).

32. Foster, P. J., Fürthauer, S., Shelley, M. J. \& Needleman, D. J. From cytoskeletal assemblies to living materials. Curr. Opin. Cell Biol. 56, 109-114 (2019).

33. Mullins, R. D. \& Hansen, S. D. In vitro studies of actin filament and network dynamics. Curr. Opin. Cell Biol. 25, 6-13 (2013).

34. Lemma, L. M. et al. Multiscale Microtubule Dynamics in Active Nematics. Phys. Rev. Lett. 127, $148001(2021)$.

35. Thijssen, K. et al. Submersed micropatterned structures control active nematic flow, topology, and concentration. Proc. Natl. Acad. Sci. 118, (2021).

36. Wu, K.-T. et al. Transition from turbulent to coherent flows in confined three-dimensional active fluids. Science 355, eaal1979 (2017).

37. Opathalage, A. et al. Self-organized dynamics and the transition to turbulence of confined active nematics. Proc. Natl. Acad. Sci. 116, 4788-4797 (2019).

38. Zhou, Z. et al. Machine learning forecasting of active nematics. Soft Matter 17, 738-747 (2021).

39. Duclos, G. et al. Spontaneous shear flow in confined cellular nematics. Nat. Phys. 14, 728-732 (2018).

40. Gompper, G. et al. The 2020 motile active matter roadmap. J. Phys. Condens. Matter 32, 193001 (2020).

41. Deneke, V. E. et al. Self-Organized Nuclear Positioning Synchronizes the Cell Cycle in Drosophila Embryos. Cell 177, 925-941.e17 (2019).

42. Lloyd, C. W. \& Traas, J. A. The role of F-actin in determining the division plane of carrot suspension cells. Drug studies. Development 102, 211-221 (1988). 
43. Yi, K. \& Li, R. Actin cytoskeleton in cell polarity and asymmetric division during mouse oocyte maturation. Cytoskelet. Hoboken NJ 69, 727-737 (2012).

44. Rasmussen, C. G., Wright, A. J. \& Müller, S. The role of the cytoskeleton and associated proteins in determination of the plant cell division plane. Plant J. 75, 258-269 (2013).

45. Seetharaman, S. et al. Microtubules tune mechanosensitive cell responses. Nat. Mater. 1-12 (2021) doi:10.1038/s41563-021-01108-x.

46. Ricketts, S. N., Ross, J. L. \& Robertson-Anderson, R. M. Co-Entangled Actin-Microtubule Composites Exhibit Tunable Stiffness and Power-Law Stress Relaxation. Biophys. J. 115, 1055-1067 (2018).

47. Francis, M. L. et al. Non-monotonic dependence of stiffness on actin crosslinking in cytoskeleton composites. Soft Matter 15, 9056-9065 (2019).

48. Ricketts, S. N. et al. Varying crosslinking motifs drive the mesoscale mechanics of actin-microtubule composites. Sci. Rep. 9, 12831 (2019).

49. Sanchez, T., Chen, D. T. N., DeCamp, S. J., Heymann, M. \& Dogic, Z. Spontaneous motion in hierarchically assembled active matter. Nature 491, 431-434 (2012).

50. Colen, J. et al. Machine learning active-nematic hydrodynamics. Proc. Natl. Acad. Sci. 118, (2021).

51. Fan, Y., Wu, K.-T., Aghvami, S. A., Fraden, S. \& Breuer, K. S. Effects of confinement on the dynamics and correlation scales in kinesin-microtubule active fluids. Phys. Rev. E 104, 034601 (2021).

52. Mitchell, K. A., Tan, A. J., Arteaga, J. \& Hirst, L. S. Fractal generation in a two-dimensional activenematic fluid. Chaos Interdiscip. J. Nonlinear Sci. 31, 073125 (2021).

53. Pandolfi, R. J., Edwards, L., Johnston, D., Becich, P. \& Hirst, L. S. Designing highly tunable semiflexible filament networks. Phys. Rev. E 89, 062602 (2014).

54. Tan, A. J. et al. Topological chaos in active nematics. Nat. Phys. 15, 1033-1039 (2019).

55. Triclin, S. et al. Self-repair protects microtubules from destruction by molecular motors. Nat. Mater. 20, 883-891 (2021).

56. Farhadi, L., Fermino Do Rosario, C., Debold, E. P., Baskaran, A. \& Ross, J. L. Active SelfOrganization of Actin-Microtubule Composite Self-Propelled Rods. Front. Phys. 6, 75 (2018).

57. Ideses, Y., Sonn-Segev, A., Roichman, Y. \& Bernheim-Groswasser, A. Myosin II does it all: assembly, remodeling, and disassembly of actin networks are governed by myosin II activity. Soft Matter 9, 71277137 (2013).

58. Cerbino, R. \& Trappe, V. Differential Dynamic Microscopy: Probing Wave Vector Dependent Dynamics with a Microscope. Phys. Rev. Lett. 100, 188102 (2008).

59. Cerbino, R., Giavazzi, F. \& Helgeson, M. E. Differential dynamic microscopy for the characterization of polymer systems. J. Polym. Sci. n/a,.

60. Germain, D., Leocmach, M. \& Gibaud, T. Differential dynamic microscopy to characterize Brownian motion and bacteria motility. Am. J. Phys. 84, 202-210 (2016). 
61. Wilson, L. G. et al. Differential Dynamic Microscopy of Bacterial Motility. Phys. Rev. Lett. 106, $018101(2011)$.

62. Stam, S. et al. Filament rigidity and connectivity tune the deformation modes of active biopolymer networks. Proc. Natl. Acad. Sci. 114, E10037-E10045 (2017).

63. The Theory of Polymer Dynamics. (Oxford University Press, 1988).

64. Gittes, F. \& MacKintosh, F. C. Dynamic shear modulus of a semiflexible polymer network. Phys. Rev. E 58, R1241-R1244 (1998).

65. On the Crawling of Animal Cells. https://www.science.org/doi/10.1126/science.8493552.

66. Porat-Shliom, N., Milberg, O., Masedunskas, A. \& Weigert, R. Multiple roles for the actin cytoskeleton during regulated exocytosis. Cell. Mol. Life Sci. CMLS 70, 2099-2121 (2013).

67. Edozie, B. et al. Self-organization of spindle-like microtubule structures. Soft Matter 15, 4797-4807 (2019).

68. McGorty, R. rmcgorty/Image-Correlation. (2020).

69. Robertson, C. \& George, S. C. Theory and practical recommendations for autocorrelation-based image correlation spectroscopy. J. Biomed. Opt. 17, 080801-080801 (2012).

70. Schindelin, J. et al. Fiji: an open-source platform for biological-image analysis. Nat. Methods 9, 676682 (2012). 


\section{Supplementary Files}

This is a list of supplementary files associated with this preprint. Click to download.

- DHAKinesinNC1221SI.pdf 